\title{
Effect of unacylated ghrelin on peripheral nerve regeneration
}

\author{
Giulia Ronchi, ${ }^{1}$ Pierluigi Tos, ${ }^{2}$ Elia Angelino, ${ }^{3}$ Luisa Muratori, ${ }^{1}$ Simone Reano, ${ }^{4}$ Nicoletta Filigheddu, ${ }^{4}$ \\ Andrea Graziani, ${ }^{3}$ Stefano Geuna, ${ }^{1}$ Stefania Raimondo ${ }^{1}$ \\ ${ }^{1}$ Department of Clinical and Biological Sciences and Neuroscience Institute of the "Cavalieri Ottolenghi" \\ Foundation (NICO), University of Turin, Orbassano (TO) \\ ${ }^{2}$ Hand Surgery and Reconstructive Microsurgery Unit, ASST G. Pini-CTO, Milan \\ ${ }^{3}$ Department of Biotechnologies and Health Sciences, Molecular Biotechnology Center, University of Turin \\ ${ }^{4}$ Department of Translational Medicine, University of Piemonte Orientale, Novara, Italy
}

Ghrelin is a circulating peptide hormone released by enteroendocrine cells of the gastrointestinal tract as two forms, acylated and unacylated. Acylated ghrelin (AG) binds to the growth hormone secretagogue receptor 1a (GHSR1a), thus stimulating food intake, growth hormone release, and gastrointestinal motility. Conversely, unacylated GHR (UnAG), through binding to a yet unidentified receptor, protects the skeletal muscle from atrophy, stimulates muscle regeneration, and protects cardiomyocytes from ischemic damage. Recently, interest about ghrelin has raised also among neuroscientists because of its effect on the nervous system, especially the stimulation of neurogenesis in spinal cord, brain stem, and hippocampus. However, few information is still available about its effectiveness on peripheral nerve regeneration. To partially fill this gap, the aim of this study was to assess the effect of UnAG on peripheral nerve regeneration after median nerve crush injury and after nerve transection immediately repaired by means of an end-to-end suture. To this end, we exploited FVB1 Myh6/Ghrl transgenic mice in which overexpression of the ghrelin gene (Ghrl) results in selective up-regulation of circulating UnAG levels, but not of AG. Regeneration was assessed by both functional evaluation (grasping test) and morphometrical analysis of regenerated myelinated axons. Results obtained lead to conclude that UnAG could have a role in development of peripheral nerves and during more severe lesions.

Key words: Ghrelin; peripheral nerve; regeneration; functional recovery; morphometry.

Correspondence: Stefania Raimondo, Department of Biological and Clinical Sciences, Neuroscience Institute Cavalieri Ottolenghi, University of Turin, Regione Gonzole 10, 10043 Orbassano (TO), Italy.

Tel. +39.011.6705433 - Fax: +39.011.9038639. E-mail: stefania.raimondo@unito.it

Contributions: SRa, SG, AG, PT, study design; SRa, SG, NF, experiment supervision; PT, surgeries; GR, functional analysis; GR, LM, SRe, morphological and morphometrical analysis; GR, SRa, EA, figure preparation and manuscript drafting; GR, PT, EA, LM, NF, AG, SG, SRa, manuscript revision and editing. All the authors have read and approved the final version of the manuscript and agreed to be accountable for all aspects of the work.

Conflict of interest: The authors declare that they have no competing interests, and all authors confirm accuracy.

Ethical Approval: All animal experiments were performed at the animal facility of Neuroscience Institute Cavalieri Ottolenghi (NICO) (Ministerial authorization DM 182 2010-A 3-11-2010). The study conditions were conformed to the guidelines of the European Union's Directive EU/2010/63 for animal experiments. 


\section{Introduction}

Peripheral nerve regeneration after a traumatic lesion is usually far from satisfactory, ${ }^{1,2}$ and there is no repair technique to guarantee total recovery and normalization of functional sensibility. Therefore, effective therapeutic measures to promote the process of peripheral nerve regeneration are necessary. Strategies for improving peripheral nerve regeneration with factor stimulation are wildly studied.

Multiple growth factors cause various regulated changes in gene or protein expression over time, ${ }^{3}$ and play a great role in the recovery of peripheral nerves after injury. A variety of neurotrophic factors were tested for the improvement of nerve regeneration. Among the various molecules that are currently being explored in experimental models with such a goal, the circulating peptide hormone ghrelin is receiving increasing interest.

Acylated ghrelin (AG) is a 28-aminoacid peptide hormone which binds to the growth hormone secretagogue receptor 1a (GHSR1a) and modulates systemic metabolism via central and peripheral actions by regulating food intake, growth hormone axis, adiposity, gut motility, and glucose metabolism. ${ }^{4}$ In addition to these functions, AG has both a regeneration promoting effect in the central nervous system ${ }^{5-7}$ and a preventive effect against skeletal muscle atrophy. ${ }^{8}$ Moreover, AG acts on peripheral nerve regeneration promoting axon and myelin repair and reducing inflammatory response after sciatic nerve injury in rats. ${ }^{9}$

High affinity binding to GHSR1a requires acylation (preferentially octanoylation) on the serine 3 residue of ghrelin peptide..$^{10,11}$ However, the unacylated form of the hormone (unacylated ghrelin - UnAG) shares with AG several GHSR1a-independent biological actions, including protection against fasting- and sciatic nerve denervation-induced skeletal muscle atrophy and sarcopenia in mice, ${ }^{8,12}$ regulation of systemic metabolism, ${ }^{13}$ and pro-survival effects on cardiomyocytes, endothelial and pancreatic $\beta$ cells. ${ }^{14,15}$ Moreover, UnAG promotes skeletal muscle regeneration in mice by acting directly on satellite cells, the muscle progenitor cells ${ }^{16}$ and $G h r l$ knockout mice exhibit impaired muscle regeneration. ${ }^{17}$

Nevertheless, while extensive studies have been conducted to unveil the role of the ghrelin/GHSR1a axis in different fields, possible effects of UnAG in peripheral nerves are not known. Our purpose was to investigate the possible effects of UnAG in promoting peripheral nerve regeneration after two different types of median nerve injury, crush and transection immediately repaired by endto-end suture.

\section{Materials and Methods}

\section{Animals and surgery}

For this study, a total of 30 adult females FVB mice weighing approximately $30 \mathrm{~g}$ were used. Animals were housed in a room with controlled temperature and humidity, with a regular light/dark circle (12 h of light and $12 \mathrm{~h}$ of dark) and free access to food and water. Every attempt was made to minimize pain and discomfort considering human endpoints for animal suffering and distress. The study conditions were conformed to the guidelines of the European Union's Directive EU/2010/63 for animal experiments. All animal experiments were performed at the animal facility of Neuroscience Institute Cavalieri Ottolenghi (NICO) (Ministerial authorization DM 182 2010-A 3-11-2010).

FVB1 Myh6/Ghrl were obtained by cloning the murine ghrelin gene $(G h r l)$ under control of the cardiac promoter sequences of the $\beta$ myosin heavy chain 3' UTR and the first 3 exons of the $\alpha$ isoform
Myh6. ${ }^{8}$ Transgene integration and expression were confirmed by PCR and real-time RT-PCR, respectively.

All surgical procedures were carried out under deep anaesthesia obtained with intramuscular injection of ketamine $(9 \mathrm{mg} / 100 \mathrm{~g}$ body weight), xylazine (1.25 mg/100 g-body weight) and atropine $(0.025 \mathrm{mg} / 100 \mathrm{~g}$-body weight). The median nerve of the left forelimb was approached from the axillary region to the elbow and carefully exposed. For the crush injury, the crush lesion was applied using a non-serrated clamp for $30 \mathrm{~s}$, as previously described. ${ }^{18}$ For the end-to-end (EtE) repair, the median nerve was transected at the level of the pectoralis major muscle and immediately repaired by means of two epineural stitches in a tension-free environment using 12-0 monofilament nylon. The 30 animals were divided in the different experimental groups as follows: 6 CTRL WT, 6 CTRL UnAG, 5 Crush WT, 5 Crush UnAG, 4 EtE WT, 4 EtE UnAG.

In order to prevent interferences with the grasping test, in all experimental groups the contra-lateral median nerve was transected at the middle third of the brachium and its proximal stump was sutured in the pectoralis major muscle to avoid spontaneous reinnervation.

Animal well-being was assessed by careful animal surveillance of passive and active movement, auto-mutilation, skin ulcers, and joint contracture.

\section{Postoperative assessment of functional recovery (grasp- ing test)}

The grasping test was performed to evaluate the functional recovery after nerve regeneration. Starting from day- 5 postoperative, functional recovery of the left forepaw was assessed every 5 days until the end of the experiment (day-25 post-operative for the crush injury groups and day-70 for the end-to-end repair groups) using the BS-GRIP Grip Meter (2Biological Instruments, Varese, Italy). Briefly, the mouse is hold by the tail and put close enough to the device to grasp it. The mouse is allowed to pull on the bar until it loses the grip. When the median nerve function is impaired, the animal's paw approaches the grid in complete finger extension. The maximum force of grasping is then recorded on the balance at the moment the animal loses the grip. The mouse is tested three times and the average value is recorded.

\section{Resin embedding and design-based stereology}

The last day of procedure (day-25 for the crush injury groups and day-70 for the end-to-end repair groups), mice were sacrificed, a 5-mm long segment of the median nerve distal to the site of lesion was removed and a $4 / 0$ stitch was used to mark the proximal stump of the nerve segment. Nerve samples were then fixed by immediate immersion in $2.5 \%$ glutaraldehyde in $0.1 \mathrm{M}$ phosphate buffer ( $\mathrm{pH} 7.4$ ) for $5-6 \mathrm{~h}$ at $4^{\circ} \mathrm{C}$. Samples were then post-fixed in $2 \%$ osmium tetroxide for $2 \mathrm{~h}$ and embedded in Glauerts' embedding mixture of resins, as previously described. ${ }^{19}$

Series of $2.5 \mu \mathrm{m}$ thick semi-thin transverse sections were cut starting from the distal stump of the withdrawn median nerve sample, using a Ultracut UCT ultramicrotome (Leica Microsystems, Wetzlar, Germany) and stained by toluidine blue. In each nerve, design-based quantitative morphology was carried out, on one randomly selected section, using a DM4000B microscope equipped with a DFC320 digital camera and an IM50 image manager system (Leica Microsystems, Wetzlar, Germany) according to the procedure described by Geuna. ${ }^{20,21}$ The following parameters were estimated: total cross-sectional area of the nerve profile, total fibre number and mean fibre density, fibre and axon diameter, myelin thickness, and axon/fibre diameter ratio, i.e. the g-ratio.

In addition, median nerves from healthy wild type and transgenic animals were harvested and analysed to detect any structural differences. 


\section{Statistical analysis}

Statistical analysis was performed using one-way analysis of variance (ANOVA) test. Statistical analysis was performed using SPSS Software. The level of significance was set at $\mathrm{p} \leq 0.05(*)$, $\mathrm{p} \leq 0.01(* *)$, and $\mathrm{p} \leq 0.001(* * *)$. Values were expressed as mean \pm Standard Deviation (SD).

\section{Results}

\section{Analysis of uninjured median nerves}

To study the possible effect of UnAG on nerve tissue, median nerves of uninjured wild type (WT) and transgenic mice (UnAG) were collected from healthy animals and analysed (Figure 1). Semithin toluidine-blue stained cross sections showed well-organized myelinated fibres with compact myelin sheaths in both experimental groups. Results of the stereological and morphometrical analysis showed a higher density and smaller myelinated fibres in UnAG group compared to WT group.

\section{Assessment of nerve regeneration after crush injury}

Functional recovery was investigated starting from day- 5 until day- 25 by means of the grasping test. The function of the finger flexor muscles innervated by the median nerve started recovering between day-10 and day-15 in both experimental groups, reaching values not significantly $(\mathrm{p}>0.05)$ different from relative pre-injured
WT and UnAG healthy nerves at day 20 (Figure 2A). No significant differences were observed between WT and UnAG regenerated groups after crush injury in any of the analysed time points.

Regenerated median nerves have been analysed with high-resolution light microscopy $5 \mathrm{~mm}$ distal to the site of crush injury. Semithin toluidine-blue stained cross sections obtained from nerves harvested 25 days after crush injury showed a good nerve regeneration in both experimental groups, as displayed in the pictures $2 \mathrm{~B}$ and $2 \mathrm{C}$. Both WT and UnAG regenerated myelinated fibres appeared smaller with a thinner myelin thickness compared to the relative uninjured controls (Figure 2D). No significant differences were observed between WT and UnAG groups in terms of number of myelinated fibers (Figure 2E), nor in the injury-induced decrease of axon and fiber diameters and myelin thickness (Figure 2F).

\section{Assessment of nerve regeneration after end-to-end repair}

The progression of functional recovery was slower in the endto-end neurorrhaphy groups in comparison to animals subjected to crush injury. Indeed, results of the grasping test showed values not significantly $(p>0.05)$ different between the two experimental groups and the relative pre-injury values starting from day 55 (Figure 3A). Moreover, UnAG animals showed higher values compared to WT animals, but this difference was significant only at day 35 .

Regenerated median nerves have been analysed with high-resolution light microscopy $5 \mathrm{~mm}$ distal to the site of end-to-end
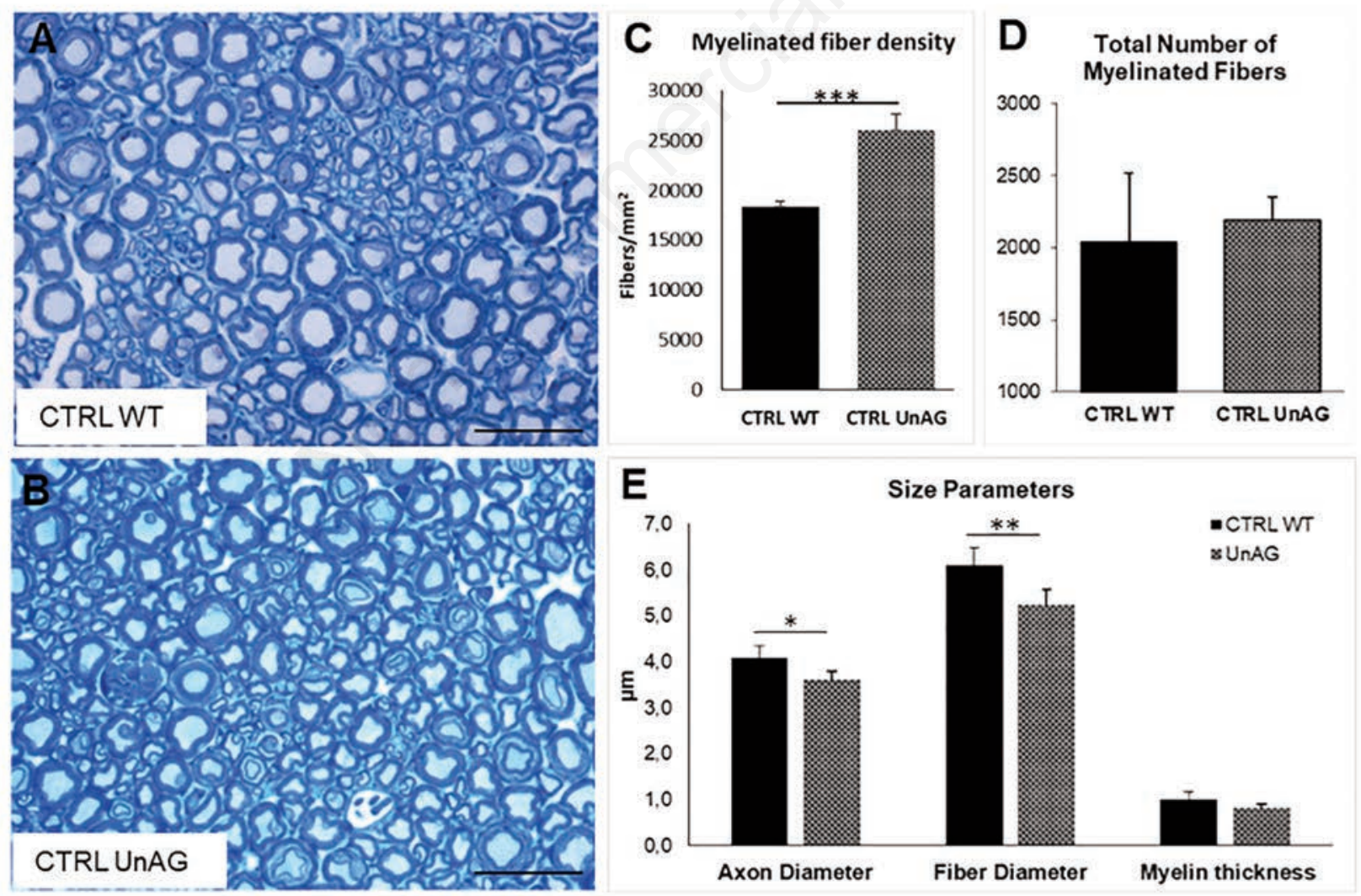

Figure 1. Results of the analysis of healthy WT and UnAG median nerves. A,B) Representative high magnification light photomicrographs of toluidine blue-stained semi-thin cross sections of healthy median nerves of WT (A) and UnAG (B) mice. C-E) Results of the stereological and morphometrical evaluation of healthy nerve fibres. C) Density of regenerated myelinated fibres. D) Total number of regenerated myelinated fibres. E) Morphometrical evaluation of size parameters: axon diameter, fiber diameter and myelin thickness. Scale bars: A,B) $20 \mu \mathrm{m}$. Values are presented as mean $\pm \mathrm{SD}$; ${ }^{*} \mathrm{p} \leq \mathbf{0 . 0 5}$; $^{*} \mathrm{p} \leq \mathbf{0 . 0 1}$; ${ }^{* *} \mathrm{p} \leq \mathbf{0 . 0 0 1}$. 
repair. At the end of the observation period (70 days post-operative), both experimental groups regenerated, as showed by semithin toluidine-blue stained cross sections (Figure $3 \mathrm{~B}, \mathrm{C}$ ). Quantitative analysis showed denser, smaller with thinner myelin thickness regenerated myelinated fibres compared to the relative uninjured controls (Figure 3 D-F).

\section{Discussion}

Ghrelin is a hormone mainly secreted from the stomach but also synthesized in a number of tissues suggesting both endocrine and paracrine effects in different functions of human body. ${ }^{22}$

However, its role in the peripheral nervous system has been poorly studied and the majority of works focus on the actions of the acylated form of ghrelin. Different studies showed the antinociceptive activity and anti-inflammatory properties of AG. ${ }^{23}$ A daily intraperitoneal administration of AG attenuated mechanical hyperalgesia after chronic constriction injury (CCI) of the rat sciatic nerve with a reduction of spinal pro-inflammatory cytokines TNF- $\alpha$ and IL-1 $\beta$ levels, but not IL-6. ${ }^{9}$ A more recent study showed a delayed thermal hyperalgesia and mechanical allodynia after intrathecal injection of AG in CCI animal with inhibition of p38 MAPK phosphorylation and the activation of NF- $\kappa B p 65$ in the spinal dorsal horn, demonstrating that ghrelin alleviated neuropathic pain through a GHSR-1a-mediated suppression of the p38 MAPK/NF- $\kappa$ B pathway. ${ }^{24}$

AG administration, but not UnAG, has been shown to prevent motor and sensory diabetic neuropathy in rodents. ${ }^{25}$ The therapeutic effects of $A G$ on diabetic peripheral neuropathy has also been demonstrated on human patients: patients treated with intravenous injection of synthetic human AG for 14 days showed improved motor nerve conduction velocity of the posterior tibial nerve and improved subjective symptoms. ${ }^{26}$

As regards the effect on nerve fibres regeneration, it has been demonstrated that 14 days of daily intraperitoneal administration of AG improves the morphology of sciatic nerve after CCI with correlated improved morphometric parameters (axon number, diameter and myelin thickness). ${ }^{9}$ Moreover, rat sciatic nerve repaired with $10 \mathrm{~mm}$ Dl-lactic- $\varepsilon$-caprolactone conduits enhanced with adipose-derived mesenchymal cells (ASCs) and AG showed
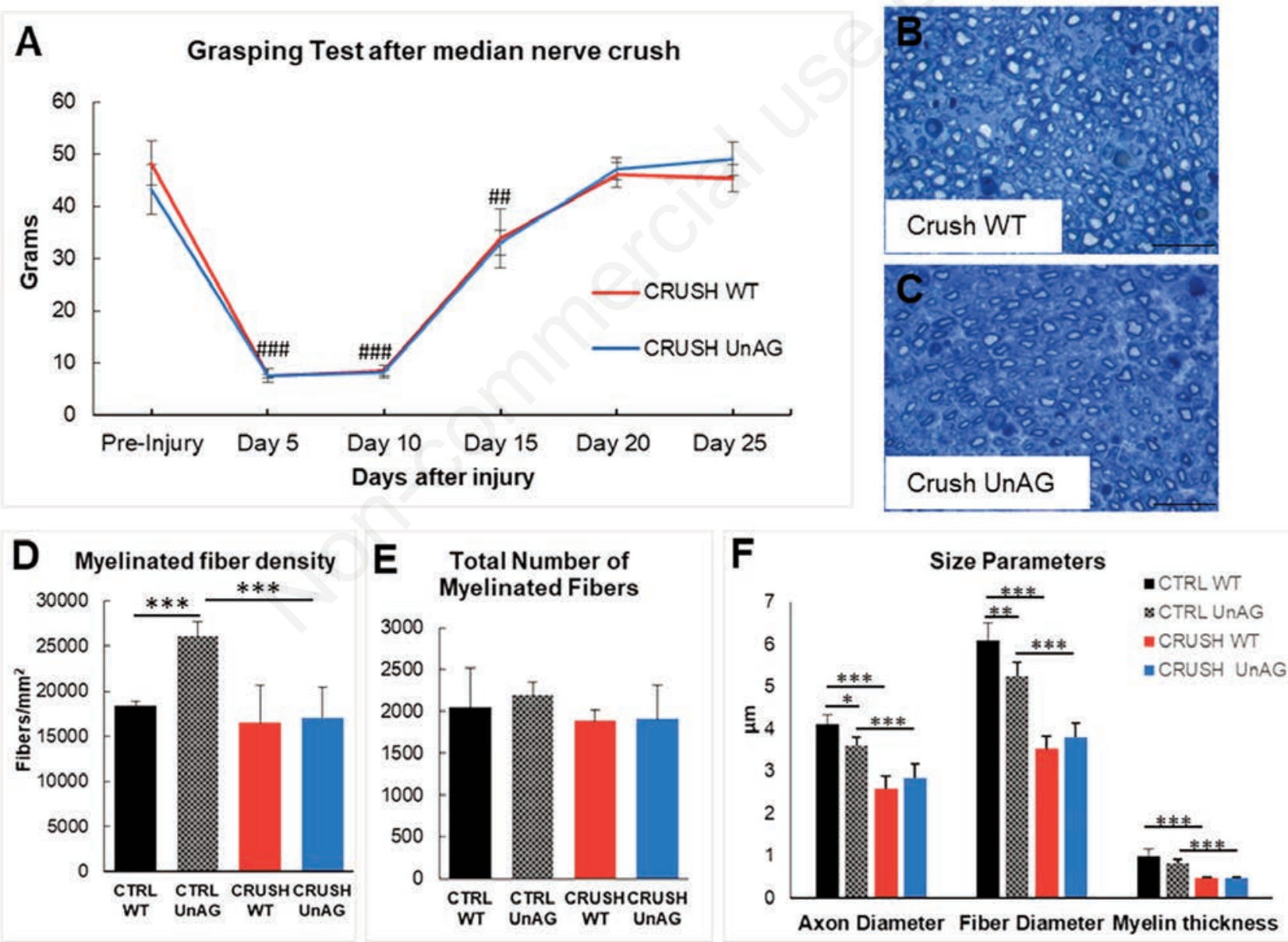
Figure 2. Results of the analysis of regenerated WT and UnAG median nerves after crush injury. A) Results of the functional recovery
of the median nerve (grasping test); \#crush WT and Crush UnAG vs relative pre-injury values; \#\# $\leq 0.01$; \#\# $\leq 0.001$. B,C)
Representative high magnification light photomicrographs of toluidine blue-stained semi-thin cross sections of the distal stump of
regenerated median nerves of WT (B) and UnAG (C) mice. D-F) Results of the stereological and morphometrical evaluation of regen-
erated nerve fibres. D) Density of regenerated myelinated fibres. E) Total number of regenerated myelinated fibres. F) Morphometrical
evaluation of size parameters: axon diameter, fiber diameter and myelin thickness. Scale bars: B,C) 20 m. Values are presented as mean
$\pm \mathrm{SD},{ }^{*} \mathrm{p} \leq \mathbf{0 . 0 5} ;{ }^{* *} \mathrm{p} \leq \mathbf{0 . 0 1}$; ${ }^{* * *} \mathrm{p} \leq \mathbf{0 . 0 0 1}$. 
a higher nerve area, myelin area and number of myelinated fibres in comparison to saline-treated control animals. ${ }^{27}$

Since UnAG, originally considered a non-functional peptide, has also proven to be active and since it represents approximately $90 \%$ of the total ghrelin detected in serum, ${ }^{28,29}$ in this study, we particularly focused our attention on the role of UnAG, using the FVB1 Myh6/Ghrl transgenic mice, in which overexpression of the ghrelin gene results in physiological levels of AG and selective upregulation of circulating UnAG.

The analysis of healthy median nerves of both WT and UnAG nerves showed that UnAG median nerves have a higher density and smaller myelinated fibres than WT nerves. These results suggest a possible role of UnAG in development that have to be deepened.

Since these differences in healthy nerves could have effects during regenerating processes, we analysed functional recovery and morphometrical parameters after two different types of injuries, crush and transection immediately repaired by EtE suture. The crush injury allows to obtain a more standardized lesion model with lower inter-individual variability in the regeneration response and a faster and more predictable functional recovery. The EtE repair of a transected nerve lead to a slower regeneration and recovery, compared to the crush lesion, due to a more severe lesion and to the microsurgical reconstruction needed for allowing regeneration. ${ }^{18}$

The data obtained in this study about functional recovery after crush injury suggest that UnAG has no effect on regeneration, as no significant differences were observed between WT and UnAG in grasping test analysis. In both groups, functional recovery starts after 15 days and reaches immediately values of control mice. Moreover, the pre-injury data showed similar values between WT and UnAG animals. These data are consistent with a previous work, where we demonstrated that WT and UnAG mice showed similar gastrocnemius muscle weight, fiber cross-sectional area distribution and hindlimb force (measured by grasping test), ${ }^{8}$ indicating that high levels of circulating UnAG do not induce skeletal muscle hypertrophy in vivo. Morphometrical analysis was performed 25 days after injury when both experimental groups showed a good nerve regeneration. Both WT and UnAG regenerated myelinated fibres appeared smaller with thinner myelin thickness compared to the relative uninjured controls. No significant differences were observed between WT and UnAG crushed groups. However, it must be considered that the healthy nerve of UnAG presents smaller fibres than healthy WT nerve, and there-
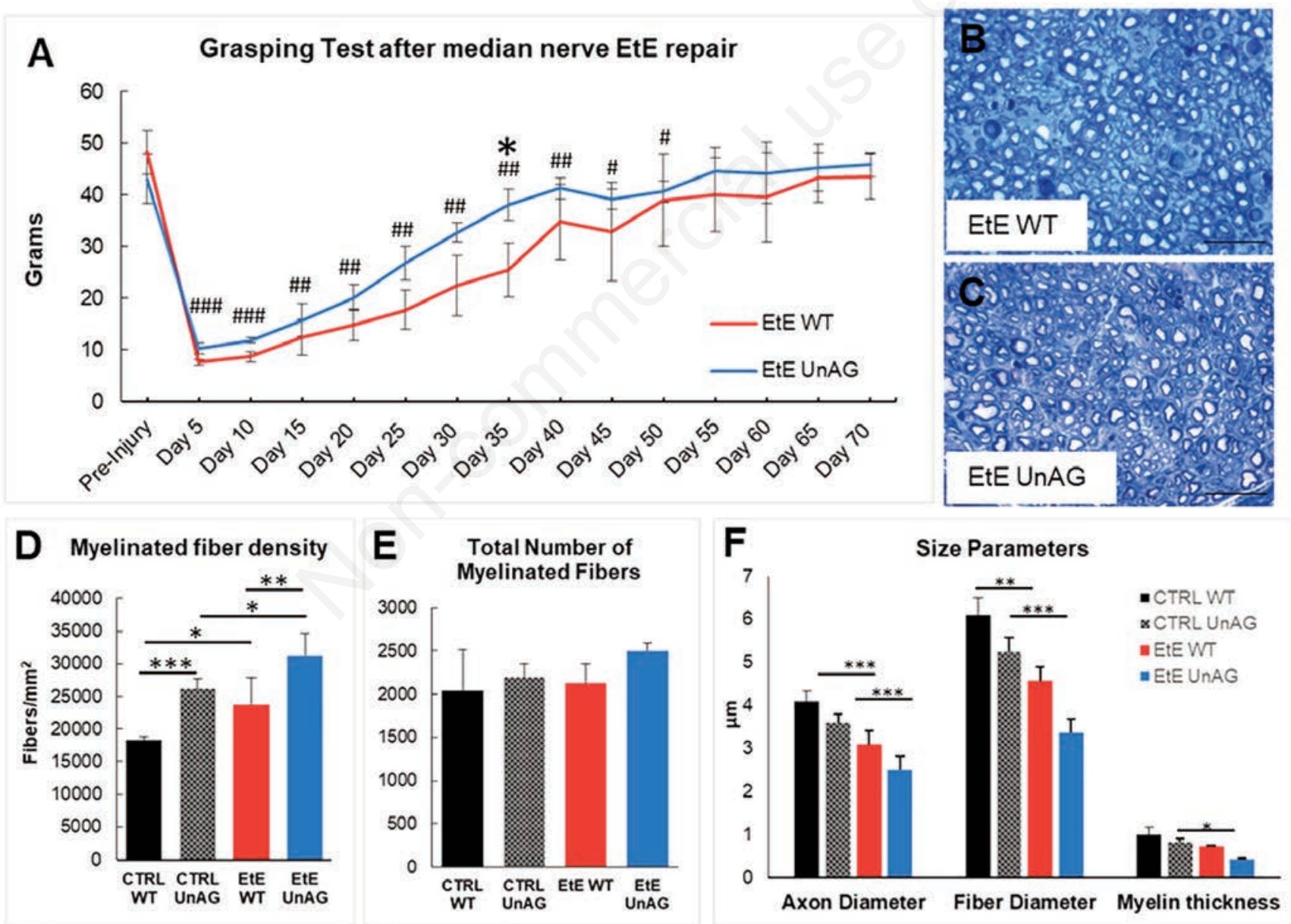

Figure 3. Results of the analysis of regenerated WT and UnAG median nerves after EtE repair. A) Results of the functional recovery of the median nerve (grasping test); ${ }^{*} \mathrm{EtE}$ WT $v$ stE UnAG; \#EtE WT and EtE UnAG vs relative pre-injury values; ${ }^{*} \mathrm{p} \leq 0.05$; \# $\leq 0.05$; \#\# $\mathbf{p} \leq \mathbf{0 . 0 1}$; \#\#\# $\leq \mathbf{0 . 0 0 1}$. B,C) Representative high magnification light photomicrographs of toluidine blue-stained semi-thin cross sections of the distal stump of regenerated median nerves of WT (B) and UnAG (C) mice. D-F) Results of the stereological and morphometrical evaluation of regenerated nerve fibres. D) Density of regenerated myelinated fibres. E) Total number of regenerated myelinated fibres. F) Morphometrical evaluation of size parameters: axon diameter, fiber diameter and myelin thickness. Scale bars: B,C) $20 \mu \mathrm{m}$. Values are presented as mean $\pm \mathrm{SD} ;{ }^{*} \mathrm{p} \leq 0.05 ;{ }^{* *} \mathrm{p} \leq 0.01 ;{ }^{* *} \mathrm{p} \leq 0.001$. 
fore the starting condition of regeneration is different.

After a more complex injury (transection) and nerve repair (EtE suture), the functional analysis showed a faster recovery in UnAG group since at day 35 values of grasping test are significantly higher than WT group. After day 55 the two experimental groups showed no significantly differences compared to CTRL demonstrating a complete functional recovery. Morphometrical analysis was performed at the end point (day 70), when functional analysis demonstrated no significant differences. This could be the reason why no significant differences between WT and UnAG EtE groups were observed in number of fibres and all size parameters.

In conclusion, results obtained on healthy nerves in this study showed a potential role of UnAG during development that has to be deeply investigated. Moreover, functional results obtained after EtE repair of the median nerve, that showed a faster recovery in UnAG EtE group in comparison to the WT group, and results obtained by others researchers that analysed the GHR effect after $\mathrm{CCI}^{9}$ and tubulisation repair, ${ }^{27}$ make us assume that UnAG could have a therapeutic role in promoting regeneration after more severe lesions.

By providing the proof of principle that UnAG promotes regeneration of injured peripheral nerves, these finding may spur further research aimed to identify the still unknown receptor mediating UnAG biological effects. The identification of the novel UnAG receptor will allow both the characterization of the mechanisms regulating its regenerative activity and the development of synthetic UnAG receptor agonists featuring pharmacological properties more suitable for therapeutic applications than UnAG which is completely metabolized 2-4 hours upon administration. ${ }^{8}$

\section{References}

1. Navarro X, Vivo M, Valero-Cabre A. Neural plasticity after peripheral nerve injury and regeneration. Prog Neurobiol 2007;82:163-201.

2. Sun W, Sun C, Zhao H, Lin H, Han Q, Wang J, et al. Improvement of sciatic nerve regeneration using laminin-binding human NGF-beta. PLoS One 2009;4:e6180.

3. Caillaud M, Richard L, Vallat JM, Desmouliere A, Billet F. Peripheral nerve regeneration and intraneural revascularization. Neural Regen Res 2019;14:24-33.

4. Muller TD, Nogueiras R, Andermann ML, Andrews ZB, Anker SD, Argente J, et al. Ghrelin. Mol Metab 2015;4:437-60.

5. Johansson I, Destefanis S, Aberg ND, Aberg MA, Blomgren K, Zhu C, et al. Proliferative and protective effects of growth hormone secretagogues on adult rat hippocampal progenitor cells. Endocrinology 2008;149:2191-9.

6. Kent BA, Beynon AL, Hornsby AK, Bekinschtein P, Bussey TJ, Davies JS, et al. The orexigenic hormone acyl-ghrelin increases adult hippocampal neurogenesis and enhances pattern separation. Psychoneuroendocrinology 2015;51:431-9.

7. Moon M, Kim S, Hwang L, Park S. Ghrelin regulates hippocampal neurogenesis in adult mice. Endocr J 2009;56:525-31.

8. Porporato PE, Filigheddu N, Reano S, Ferrara M, Angelino E, Gnocchi VF, et al. Acylated and unacylated ghrelin impair skeletal muscle atrophy in mice. J Clin Invest 2013;123:611-22.

9. Guneli E, Onal A, Ates M, Bagriyanik HA, Resmi H, Orhan $\mathrm{CE}$, et al. Effects of repeated administered ghrelin on chronic constriction injury of the sciatic nerve in rats. Neurosci Lett 2010;479:226-30.

10. Yang J, Brown MS, Liang G, Grishin NV, Goldstein JL. Identification of the acyltransferase that octanoylates ghrelin, an appetite-stimulating peptide hormone. Cell 2008;132:387-96.

11. Kojima M, Hosoda H, Date Y, Nakazato M, Matsuo H,
Kangawa K. Ghrelin is a growth-hormone-releasing acylated peptide from stomach. Nature 1999;402:656-60.

12. Agosti E, De Feudis M, Angelino E, Belli R, Alves Teixeira M, Zaggia I, et al. Both ghrelin deletion and unacylated ghrelin overexpression preserve muscles in aging mice. Aging (Albany NY) 2020;12:13939-57.

13. Delhanty PJ, Sun Y, Visser JA, van Kerkwijk A, Huisman M, van Ijcken WF, et al. Unacylated ghrelin rapidly modulates lipogenic and insulin signaling pathway gene expression in metabolically active tissues of GHSR deleted mice. PLoS One 2010;5:e11749.

14. Baldanzi G, Filigheddu N, Cutrupi S, Catapano F, Bonissoni S, Fubini A, et al. Ghrelin and des-acyl ghrelin inhibit cell death in cardiomyocytes and endothelial cells through ERK1/2 and PI 3-kinase/AKT. J Cell Biol 2002;159:1029-37.

15. Granata R, Settanni F, Biancone L, Trovato L, Nano R, Bertuzzi F, et al. Acylated and unacylated ghrelin promote proliferation and inhibit apoptosis of pancreatic beta-cells and human islets: involvement of 3',5'-cyclic adenosine monophosphate/protein kinase A, extracellular signal-regulated kinase 1/2, and phosphatidyl inositol 3-Kinase/Akt signaling. Endocrinology 2007;148:512-29.

16. Reano S, Angelino E, Ferrara M, Malacarne V, Sustova H, Sabry O, et al. Unacylated ghrelin enhances satellite cell function and relieves the dystrophic phenotype in Duchenne muscular dystrophy mdx model. Stem Cells 2017;35:1733-46.

17. Angelino E, Reano S, Bollo A, Ferrara M, De Feudis M, Sustova H, et al. Ghrelin knockout mice display defective skeletal muscle regeneration and impaired satellite cell selfrenewal. Endocrine 2018;62:129-35.

18. Ronchi G, Raimondo S, Varejao AS, Tos P, Perroteau I, Geuna S. Standardized crush injury of the mouse median nerve. J Neurosci Methods 2010;188:71-5.

19. Raimondo S, Fornaro M, Di Scipio F, Ronchi G, GiacobiniRobecchi MG, Geuna S. Chapter 5: Methods and protocols in peripheral nerve regeneration experimental research: part IImorphological techniques. Int Rev Neurobiol 2009;87:81-103.

20. Geuna S. Appreciating the difference between design-based and model-based sampling strategies in quantitative morphology of the nervous system. J Comp Neurol 2000;427:333-9.

21. Geuna S, Tos P, Battiston B, Guglielmone R. Verification of the two-dimensional disector, a method for the unbiased estimation of density and number of myelinated nerve fibers in peripheral nerves. Ann Anat 2000;182:23-34.

22. Korbonits M, Goldstone AP, Gueorguiev M, Grossman AB. Ghrelin--a hormone with multiple functions. Front Neuroendocrinol 2004;25:27-68.

23. Fields D, Miranpuri S, Miranpuri G, Resnick D. The multifunctional and multi-system influence of Ghrelin in the treatment of diabetic and spinal cord injury induced Neuropathy. Ann Neurosci 2011;18:118-22.

24. Zhou CH, Li X, Zhu YZ, Huang H, Li J, Liu L, et al. Ghrelin alleviates neuropathic pain through GHSR-1a-mediated suppression of the $\mathrm{p} 38 \mathrm{MAPK} / \mathrm{NF}-\mathrm{kappaB}$ pathway in a rat chronic constriction injury model. Reg Anesth Pain Med 2014;39:137-48.

25. Tsuchimochi W, Kyoraku I, Yamaguchi H, Toshinai K, Shiomi $\mathrm{K}$, Kangawa K, et al. Ghrelin prevents the development of experimental diabetic neuropathy in rodents. Eur J Pharmacol 2013;702:187-93.

26. Ueno H, Shiiya T, Nagamine K, Tsuchimochi W, Sakoda H, Shiomi K, et al. Clinical application of ghrelin for diabetic peripheral neuropathy. Endocr J 2017;64:S53-7.

27. Hernandez-Cortes P, Toledo-Romero MA, Delgado M, Gonzalez-Rey E, Gomez Sanchez R, Prados-Olleta N, et al. 
Ghrelin and adipose-derived mesenchymal stromal cells improve nerve regeneration in a rat model of epsilon-caprolactone conduit reconstruction. Histol Histopatho. 2017;32:627-37.

28. Asakawa A, Inui A, Fujimiya M, Sakamaki R, Shinfuku N, Ueta $\mathrm{Y}$, et al. Stomach regulates energy balance via acylated ghrelin and desacyl ghrelin. Gut 2005;54:18-24.
29. Delhanty PJ, Neggers SJ, van der Lely AJ. Mechanisms in endocrinology: Ghrelin: the differences between acyl- and desacyl ghrelin. Eur J Endocrinol 2012;167:601-8.

Received for publication: 7 June 2021. Accepted for publication: 13 August 2021.

This work is licensed under a Creative Commons Attribution-NonCommercial 4.0 International License (CC BY-NC 4.0).

(C) Copyright: the Author(s), 2021

Licensee PAGEPress, Italy

European Journal of Histochemistry 2021; 65(s1):3287

doi:10.4081/ejh.2021.3287 